\title{
Forming state recognition in deep drawing process with machine learning
}

\author{
Tomohiro TSURUYA*, Musashi DANSEKO**, Katsuhiko SASAKI***, Shinya HONDA*** \\ and Ryo TAKEDA*** \\ *Industrial Research Institute, Hokkaido Research Organization \\ Kita19 Nishi11, Kita-ku, Sapporo, Hokkaido 060-0819, Japan \\ E-mail: tsuruya-tomohiro@hro.or.jp \\ ${ }^{* *}$ Graduate School of Engineering, Hokkaido University \\ Kita 13 Nishi 8, Kita-ku, Sapporo, Hokkaido 060-8628, Japan \\ ${ }^{* * *}$ Faculty of Engineering, Hokkaido University \\ Kita 13 Nishi 8, Kita-ku, Sapporo, Hokkaido 060-8628, Japan
}

Received: 8 April 2019; Revised: 1 August 2019; Accepted: 4 September 2019

\begin{abstract}
In press processing, quality inspection of a product is often carried out for each lot in the post process stage. When a failure occurs, it may result in a large number of defective products due to the fast processing speed. In order to prevent this, it is ideal to immediately stop the processing just after the defect occurs. Therefore, confirming the processing state in-process is required. This study proposes a new quality inspection method for deep drawing processes by using the count rate of acoustic emission (AE) signals. To analyze the AE count, deep learning, which is a multilayered neural network, is employed to recognize defects during a deep drawing process. The material used was a ductile material of cold rolled steel plate and is relatively difficult to find cracks during the deep drawing process. Characteristics were clarified by analysis of $\mathrm{AE}$ counts at plastic deformation and brake of material, and performing the forming state recognition experiment by a multilayer neural network (deep learning) showed a maximum recognition rate of $97.3 \%$. High recognition rate was obtained despite the small number of data used.
\end{abstract}

Keywords : Sheet metal forming, Acoustic emission, Deep learning, Deep drawing, Data acquisition, Sensor

\section{Introduction}

Sheet metal forming is widely used as a mass production processing technology, mainly in the automobile industry, due to its high processing speed and low processing cost compared with other processing methods. In the production site of sheet metal forming, sampling inspections are usually conducted for each lot in the post-process stage. This inspection in the post-process leads to a large number of defective products due to the high processing speed. In order to suppress this, studies such as press motion control (Tamai et al., 2014), blank holding force control (Kitayama et al., 2010 and 2018), prediction of defect occurrence by simulation (Lal et al., 2018), quality evaluation of products (Müller et al., 2018), quality prediction of products, and optimization of forming conditions (Rodrigues et al., 2010) have been reported. However, in order to avoid large number of defects, it is ideal to immediately stop the processing just after the defect product is discovered. Therefore, a monitoring system for the forming state in-process is required.

There are some previous studies that monitored the forming state in-process using ultrasonic waves, die deformation / forming loads, and acoustic emissions (AEs). As for the ultrasonic waves, the monitoring of the in-process detection of wrinkles caused by the deep drawing was conducted using the relative reflection intensity of ultrasonic waves measured from a metal die embedded ultrasonic probe (Kakinoki et al., 2017). Also, ultrasonic reflection / transmission wave height ratio was used to detect the contact states between the die and product during in-process sheet metal forming (Hagino et al., 2013 and 2016). Moreover, reflected ultrasonic waves were used to determine the geometry of the material within 
the die during hydroforming (Keitmann-Curdes et al., 2004). However, there are some issues that need to be addressed when using ultrasonic waves. One major issue is that previous reports using ultrasonic waves did not consider cracks during the forming state. The other is the fact that modification to the die is required for mounting the ultrasonic sensors, thus leading to change in the rigidity of the die and in the worst case scenario reduce the processing accuracy of the product.

Measurement methods for the die deformation and forming load has been reported as well. A measurement method of the bending angle of a die was proposed using a semiconductor strain gauge incorporated into a die (Koyama and Yang, 2010). In addition, a detection method of cracks and wrinkles in deep drawing using both the punch load and the blank holder load obtained from both a sensor embedded in the die and images captured by a camera was developed by Garcia (2005). Mahayotsanun et al. proposed a method using the on-line process monitoring during sheet metal forming to measure the draw-in amount of the sheet metals, and the contact pressure on the tool-workpiece interface (Mahayotsanun et al., 2009). In the case of using the deformation / forming load of the die, again the modification on the die itself is required.

Methods using AE technology has been reported as a countermeasure for this modification to the die. AE technology is advantageous to conventional means due to the fact that $\mathrm{AE}$ sensors do not require direct contact to the die itself. AE signals were used to identify conditions of occurring cracks and lubrication, and material differences in deep drawing process using both the maximum amplitude and number of generations (Behrens et al., 2017). Using the maximum amplitude of AE signals in the blanking, conditions of burr occurrence was discussed (Nishimoto et al., 2005). Also, conditions of crack formation in automotive press panel was explored using the duration of the AE signal (Yoo et al., 2016). The root mean square of amplitude of the AE signal was used to monitor the initial deformation state of material in cup-drawing (Hao et al., 2000). A friction source detection system using multi-acoustic emission sensors and a data fusion system was applied for measuring the friction during deep drawing of the sheet metal (Yang et al., 2003). It has been shown that AE signals include useful information according to the material conditions. However, the AE signal are easily affected by external noise and thus creates a problem to identify the emitting source of the signal. This means repeatability is a major issue, and huge amounts of AE signal features must be collected to differentiate those caused by a target of interest (cracks, defects, etc.) and those from background noise. Therefore, a data processing method for accurately recognizing defective products must be established. Moreover, identification of cracks is difficult during the deep drawing process of the ductile material such as cold rolled steel plates.

Recently, image recognition technologies based on the machine learning are developing rapidly and are being applied to various fields. For example, Madokoro et al. (2016) conducted the recognition of posture on the bed by a load sensor, Masuda et al. (2017) distinguished the species of bats by ultrasonic sound, Pham (2018) worked on the spatial prediction of landslides, and Ding (2017) developed a spindle bearing fault diagnosis via vibration signals. However, there are few reports that adopt machine learning for identifying forming states.

This paper investigates a new forming state evaluation method by machine learning using multilayered neural networks based on the AE count obtained during deep drawing process on ductile cold rolled steel plates. First, the basic data on the AE count during tensile deformation was obtained using a typical specimen to clarify a difference between the AE count generated during the plastic deformation and the point of break. Measurements of the AE count during the deep drawing process are carried out for the next step. In the deep drawing process, we conducted a recognition experiment of forming state by machine learning using AE counts and confirmed its effectiveness.

\section{Experimental method}

\subsection{Tensile test}

In recognizing the forming state, it is necessary to obtain the basic data of AE signal during tensile deformation using a typical specimen. Therefore, a tensile test is performed by attaching an AE sensor to the material. The AE signal is measured until the specimen fails.

The material for the specimen is cold rolled steel plate, the shape of the tensile test piece is a strip ( $300 \mathrm{~mm}$ long, 40 $\mathrm{mm}$ wide and $1.0 \mathrm{~mm}$ thick), and the test speed is $30 \mathrm{~mm} / \mathrm{min}$. An AE sensor with a resonance frequency of $70 \mathrm{kHz}$ was attached to the specimen with a magnetic holder. The AE signal input to the measuring device is automatically amplified with a gain value of 20 times and processed with a high-pass filter with a threshold value of $100 \mathrm{kHz}$. The signal exceeding $0.1 \mathrm{v}$ is input to the mono-multi vibrator by the comparator, output to the rate meter at $1 \mathrm{msec}$ cycle, and converted to the generation rate per second (count rate). Finally, the count rate was recorded on a PC via an analog input device at a 
sampling time of $10 \mathrm{msec}$. The count rate is usually used as a parameter for a "burst type" data processing (Hatano, 1975).

Figures 1 and 2 show the experimental set-up and the schematic illustration of the data acquisition system for AE, respectively. Data were recorded around the unstable state in the stress-strain relationship and around the break of the specimen.

\subsection{Deep drawing test}

In the deep drawing process, the $\mathrm{AE}$ signal includes the signal which comes from both contact and friction between the work piece and die. This means that the AE signal obtained in the deep drawing process maybe different from that of

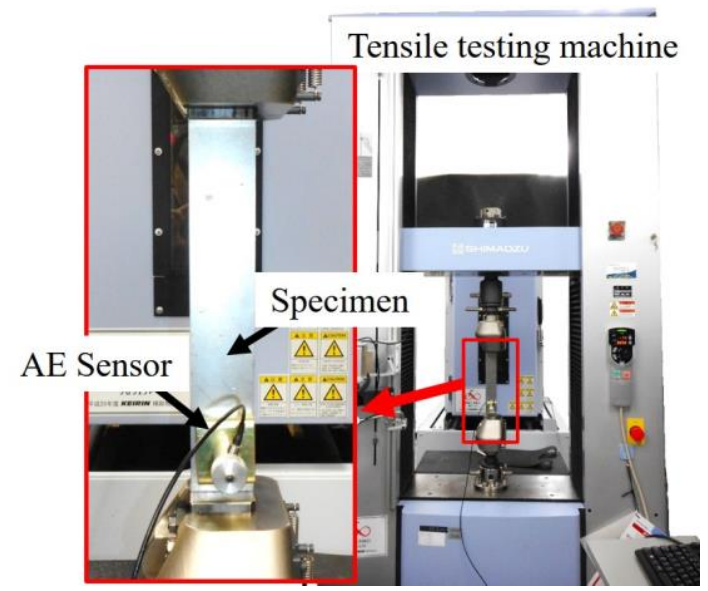

Fig. 1 Experimental set-up to analyze and measure the AE that occurs when the state of the target material changes. The material is cold rolled steel plate (nominal thickness $1.0 \mathrm{~mm}$ ), the shape of the tensile test piece is a strip. An AE sensor with a resonance frequency of $70 \mathrm{kHz}$ was used and attached to the material with a magnetic holder

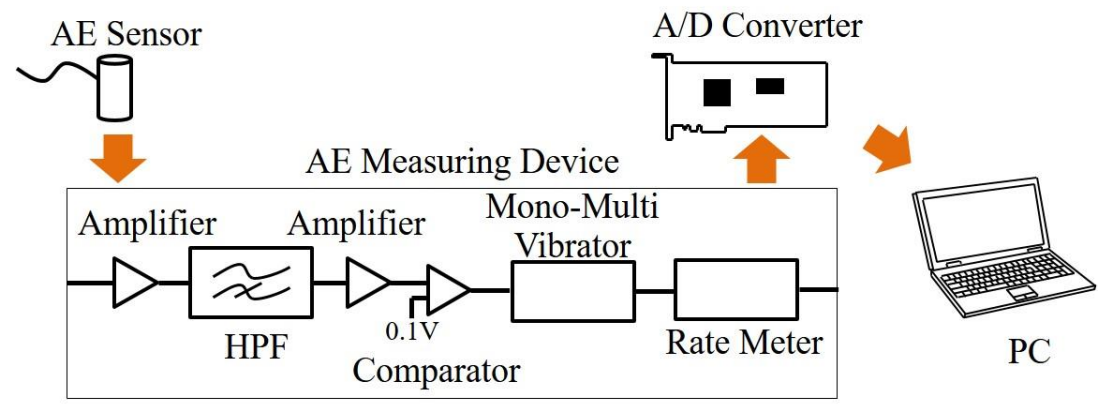

Fig. 2 Schematic illustration of the data acquisition system for AE. The AE signal is amplified by a measuring device and subjected to high-pass filtering with a threshold value of $100 \mathrm{kHz}$, counted the count rate of AE signals that exceed $0.1 \mathrm{~V}$, and recorded on a PC via an analog input device. The AE measuring device used is AE9501A (NF corporation).

Table 1 Experimental conditions for the deep drawing test.

\begin{tabular}{l|l}
\hline \hline Apparatus & Mechanical servo press \\
\hline Nominal capacity & $800 \mathrm{kN}$ \\
\hline Stroke & $160 \mathrm{~mm}$ \\
\hline Blank holder force & $6 \mathrm{kN}$ \\
\hline Shape of drawing & Circular cup \\
\hline Blank diameter & $110 \mathrm{~mm}$ \\
\hline Punch diameter & $52 \mathrm{~mm}$ \\
\hline Depth of draw & $37 \mathrm{~mm}$ \\
\hline Clearance & $1 \mathrm{~mm}$ \\
\hline
\end{tabular}


the signal during the tensile deformation. Therefore, measurement of the AE signal during the deep drawing process are carried out and the AE count is compared with those obtained during the tensile tests.

The work piece used here is made of the same material of cold rolled steel plate (nominal thickness $1.0 \mathrm{~mm}$ ) as the tensile testing. Experimental conditions in deep drawing are shown in Table 1. In order to obtain accurate AE signals during the deep drawing, the AE sensor was attached by a magnetic holder to the punch plate, with a large contact area to the material as shown in Fig. 3. Figure 3 shows the forming machine and mounting location of AE sensor for measurement of the AE signal during the deep drawing test. Figure 4 shows the schematic illustrations of the experimental setup and the deep drawing press. An inverted die is used, punch and die positions are interchanged. In order to accurately measure the AE signal during the deep drawing test, a single AE sensor was attached by a magnetic holder to the punch plate, that has a large contact area with the material. The press load is measured with a load cell built into the press machine to compare AE data with the forming status. The sheet metal is restrained by the blank holder and the center part is pushed into the die with the punch.

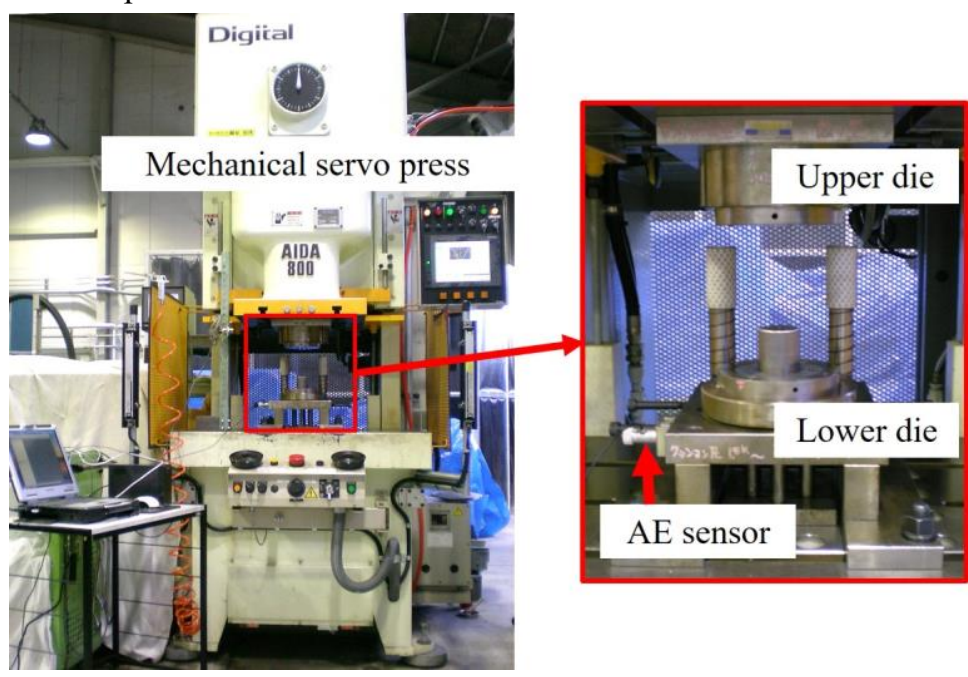

Fig. 3 Forming machine and mounting location of AE sensor for measurement and analysis of the AE signal during the deep drawing test.

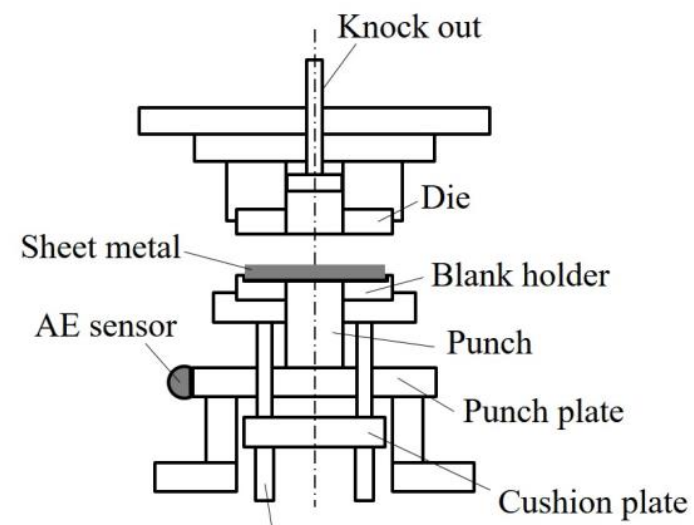

Cushion pin

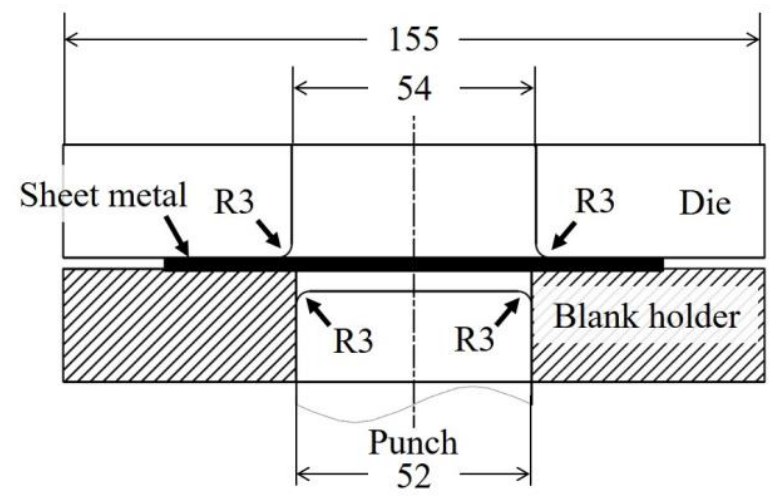

Dimensions in $\mathrm{mm}$

(a) Schematic illustration of the experimental setup

(b) Schematic illustration of the deep drawing process

Fig. 4 (a) Schematic illustration of the experimental setup. The material used is the same cold rolled steel plate (nominal thickness $1.0 \mathrm{~mm}$ ) as the tensile testing. Experimental conditions in deep drawing are shown in Table 1. In order to more accurately measure the AE signal during the deep drawing test, a single AE sensor was attached by a magnetic holder to the punch plate, that has a large contact area with the material. (b) Schematic illustration of the deep drawing process. The sheet metal is restrained by the blank holder and the center part is pushed into the die with the punch. 
Figure 5 shows punch speed curves used in this study. Since this study employed a mechanical servo press, the horizontal axis shows the crank angle. The crank angle of 180 degrees indicates bottom dead center, the crank angle of 0 and 360 degrees indicates top dead center. At the dead point the punch speed is zero, and at the crank angle of around 90 degrees and 270 degrees the punch speed maximum.

Normal products and products with cracks can be obtained with changing the amount of lubricating oil during forming. In the experiment, the lubricating oil is applied to the first specimen with a brush, and the forming of subsequent specimen continues without lubrication until cracking occurs. After the occurrence of cracks, lubricant is again applied with a brush on the first sheet, and then forming without lubrication continued until cracking reoccurs. This process is repeated until 100 specimens are formed.

\subsection{Recognition of deep drawing state by machine learning}

The AE data from the deep drawing testing system shown in Fig. 3 was used as the input (learning) data for the machine learning method. The AE signal was amplified by a tester and subjected to a high-pass filter with a threshold value of $100 \mathrm{kHz}$. The count rate counted by the comparator was recorded on a PC via an analog input device at a sampling time of $10 \mathrm{msec}$. The number of data points per forming was set to 200 and 100 learning data (61 normal forming data and 39 cracking data) were collected. The proximity switch, the fact that the slide of the press lowered to a

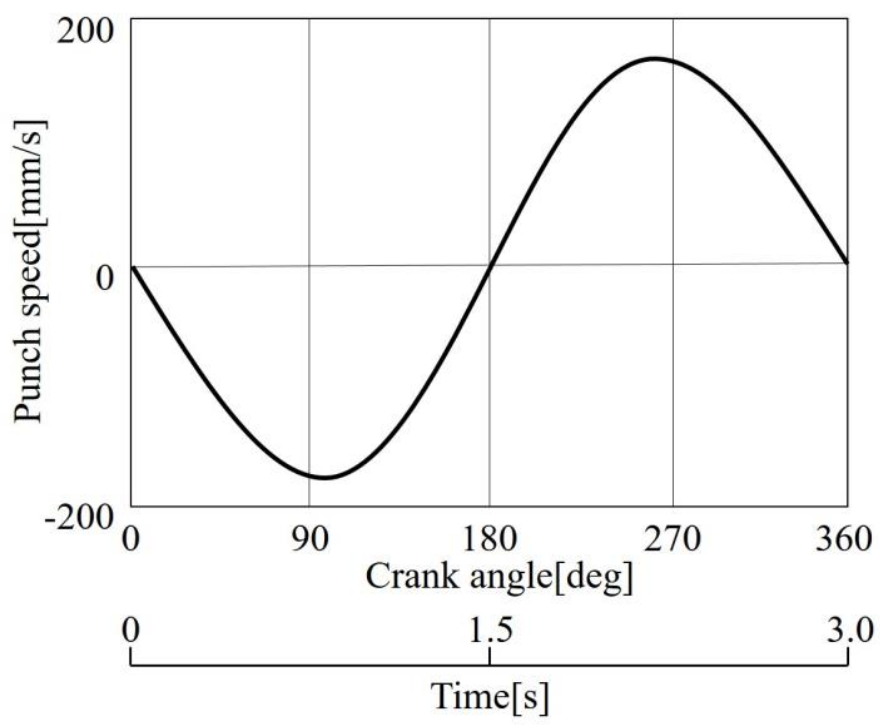

Fig. 5 Punch speed curves used in this study.

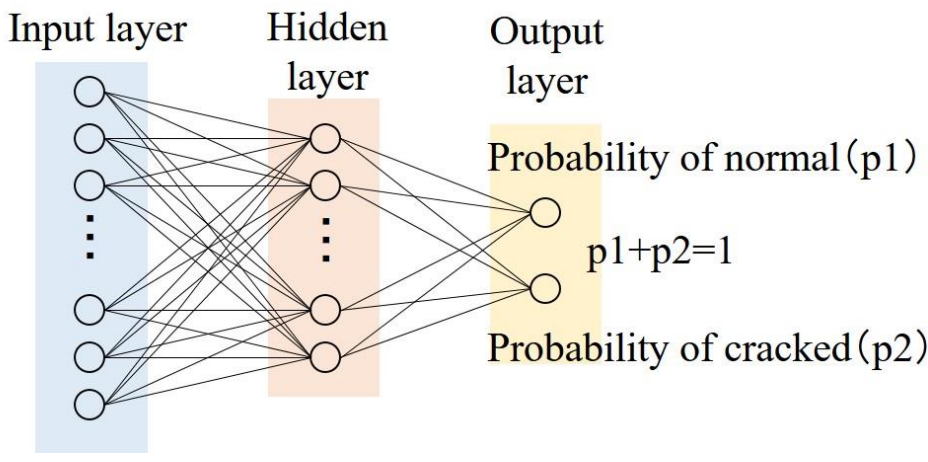

Fig. 6 The configuration of the network. Each layer is fully a connected layer, the number of nodes in the input layer is the same as the number of data points per forming, and the number of nodes in the output layer is set to 2 , in order to output the probability of normal forming and cracking. The count rate is inserted into each node of the input layer one by one. 
predetermined position, was used as a uniform trigger for starting the data acquisition. A forming state recognition system was constructed using an open source machine learning framework called "Chainer". This framework designs, learns, and evaluates the neural networks based on Python provided by Preferred Networks Co., Ltd. It also has features such as simultaneous execution of network construction and evaluation, use of GPU, high speed calculation, use of various loss functions and optimizers, and easy and flexible description of multiple layers and complicated networks.

The configuration of the network is shown in Fig.6. Each layer is a fully connected layer, the number of nodes in the input layer is the same as the number of data points per forming, and the number of nodes in the output layer is set to 2 in order to output the probability of the normal forming and cracking. The count rate is inserted into each node of the input layer one by one. The probabilities of the normal forming and cracking were compared and the forming state with

Table 2 Computer specifications, software version and training time of the machine learning.

\begin{tabular}{l|l}
\hline \hline CPU & Intel Xeon E3-1241 V3 3.50 GHz, 4 cores 8 threads \\
\hline Memory size & 16 GB DDR3, Non-ECC \\
\hline GPU & NVIDIA Quadro K620, 2 GB DDR3 \\
\hline OS & Ubuntu 14.04.5 LTS \\
\hline Versions of Chainer & V1 \\
\hline Training time & 1.17 s (average time of three trials.) \\
\hline
\end{tabular}

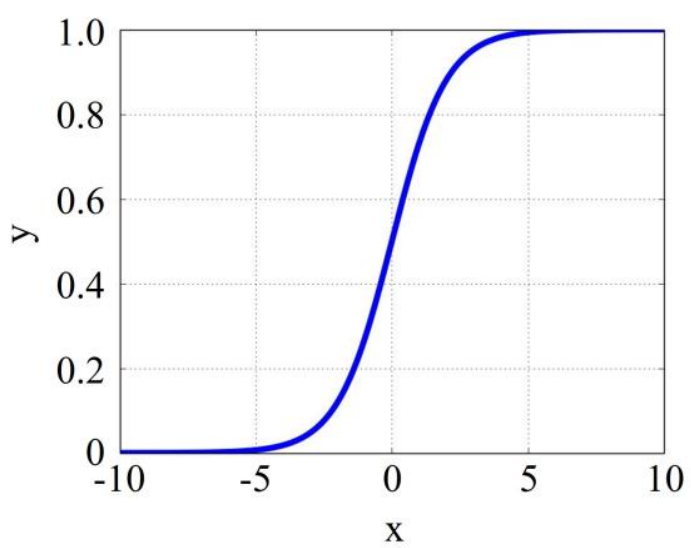

(a) Sigmoid

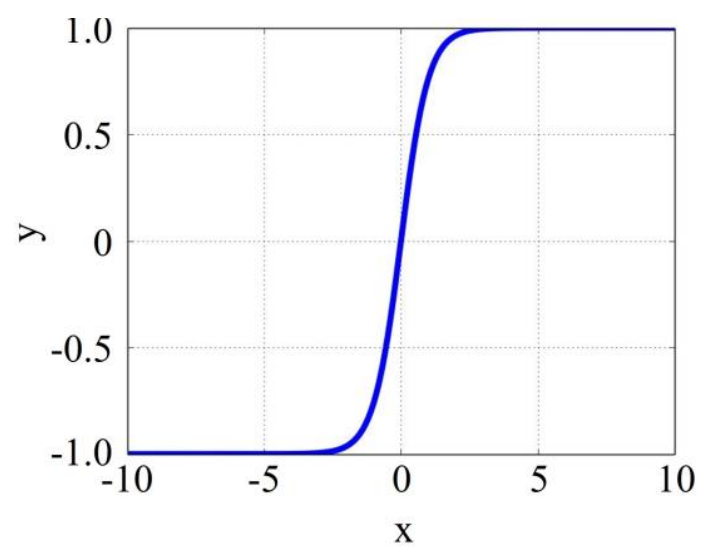

(b) $\tanh$

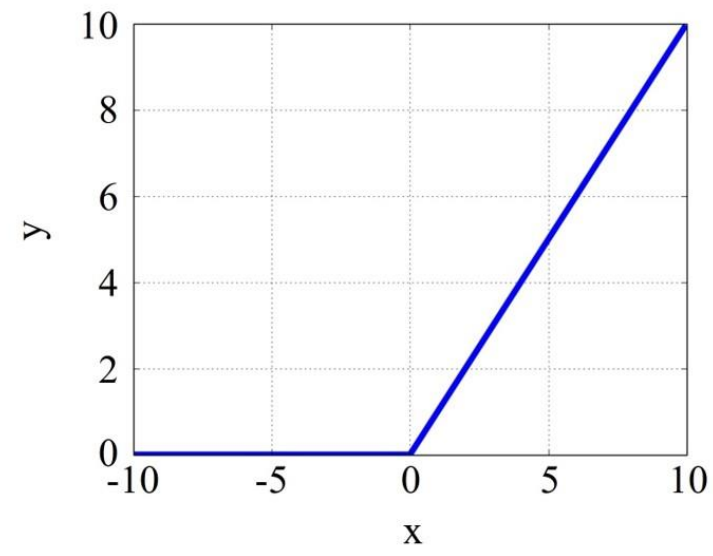

(c) ReLU

Fig. 7 Activation functions. (a) Sigmoid, (b) tanh, and (c) ReLU. 
the larger probability was judged as the forming state of the input data. 80 randomly selected from 100 data sets were set as the learning data. The learning process using 10 data sets randomly selected from the learning data was repeated 30 times. Finally, using the learned model, the forming state recognition experiment was performed by changing the number of nodes in the middle layer, the optimization method, and the activation function for all 100 data sets. Three optimization methods, SGD (Stochastic Gradient Descent), RMSprop (Root Mean Squared propagation), and Adam (Adaptive moment estimation) and three activation functions ReLU (Rectified Linear Unit), tanh, and sigmoid were used. SGD is an optimizer for randomly selected data. RMSprop is an optimizer by exponential moving average which gradually reduces influence of past information. Adam is an optimizer that cancels bias due to exponential moving average generated by RMSprop. RMSProp is a robust to non-stationary problem settings, and Adam achieves optimal convergence performance with many problem settings. SGD is the most basic method and is used for comparison. Computer specifications and software version are shown in Table 2.

As for Sigmoid, the output value y approaches to 1 from 0 with increase in the input value $x$ as shown in Fig. 7 (a) and Eq. (1). As for tanh, the output value y approaches to 1 from -1 with increase in the input value $\mathrm{x}$ as shown in Fig. 7 (b) and Eq. (2). As for ReLU, the output value $\mathrm{y}$ is 0 when the input value $\mathrm{x}$ is negative, while the output value $\mathrm{y}$ linearly increases when the input value $\mathrm{x}$ is positive as shown in Fig. 7 (c) and Eq. (3). In the conventional neural network, sigmoid and tanh are mainly used, but it takes time for calculation and it may become impossible to calculate if there are many layers. ReLU is used because the calculation speed is high and is effective even if there are many layers. In this paper, nine combinations of the three activation functions (Sigmoid, tanh, and ReLU) and the three optimization methods (SGD, RMSprop, and Adam) are used for the recognition.

$$
\begin{aligned}
& y=\frac{1}{1+\exp (-x)} \\
& y=\frac{\exp (x)-\exp (-x)}{\exp (x)+\exp (-x)} \\
& y=\max (0, x)
\end{aligned}
$$

\section{Results and discussions}

\subsection{AE characteristic in tensile test}

Figure 8 shows the stress-strain relation obtained by the tensile test. Dashed lines in Fig. 8 show the location where the AE count was used for the analysis. Two locations are chosen for the AE count; around the peak stress and the point

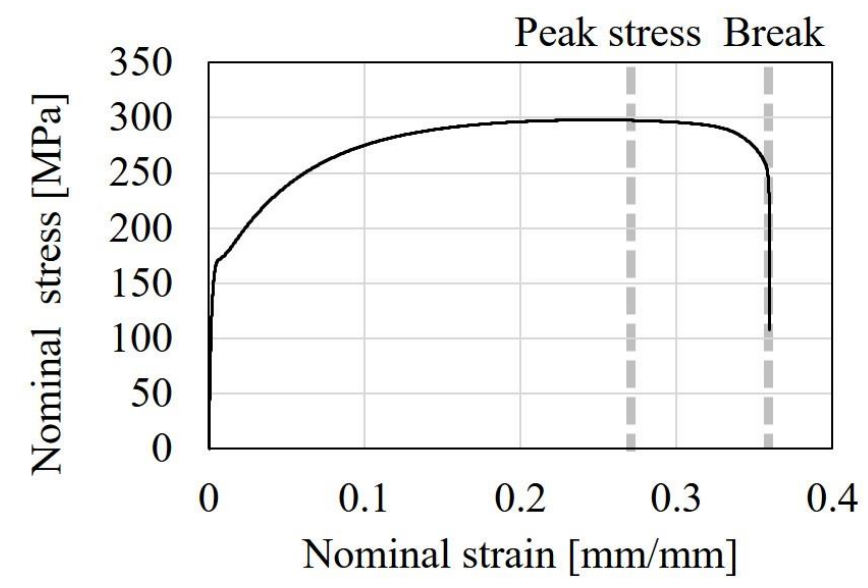

Fig. 8 Detailed analysis points for tensile tests. Recording of data was carried out overall. The analysis was performed at two points around the peak stress where plastic deformation has proceeded past the elastic deformation region and at the point of break. 
of break. Figure 9 shows the AE count obtained by the tensile test. The vertical axis is the count rate, and the horizontal axis time. Figure 9 shows the AE count rate overall, the peak stress and the occurrence of break, respectively. Figure 9 (a) shows the AE count rate at the transition from elastic deformation to plastic deformation is relatively large, it becomes smaller at the peak stress and becomes larger again at the break. Figure 9 (b) indicate that there are many peaks. Figure 9 (c) indicate that there is one big peak. AE signals are continuously generated during plastic deformation and occurs intensively at the time of break. As Kishi et al. (1977) suggested, these results indicate that continuous AE signals are dominant during plastic deformation and material breaking leads to short bursts of AE signals. There is a clear difference between the AE signal count generated during the plastic deformation around the peak stress and the point of break. Therefore, this indicates that it is possible to recognize the occurrence of cracking of material using AE counts.

\subsection{AE characteristic in deep drawing test}

Figure 10 shows products of deep drawing. From the comparison of the workpieces in Fig. 10, the flange area of cracked product is larger than the flange areas of normal product. As a result, it can be confirmed that cracks occurred at

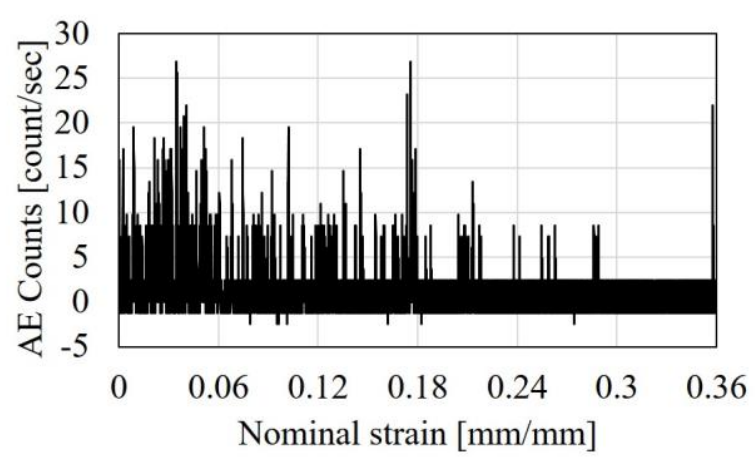

(a) overall

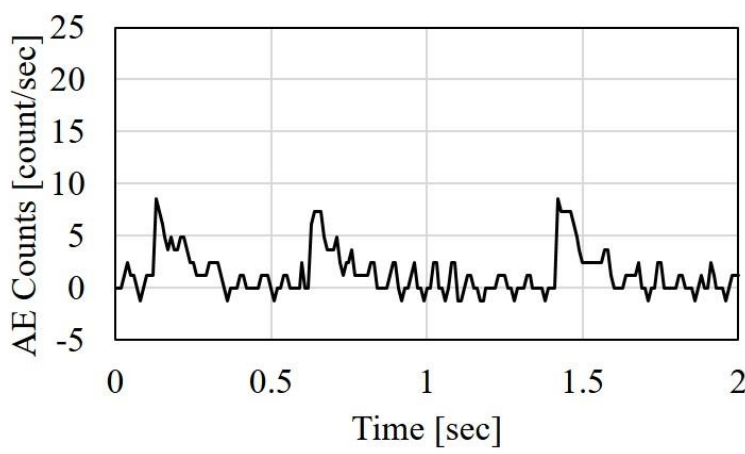

(b) time of plastic deformation around the peak stress

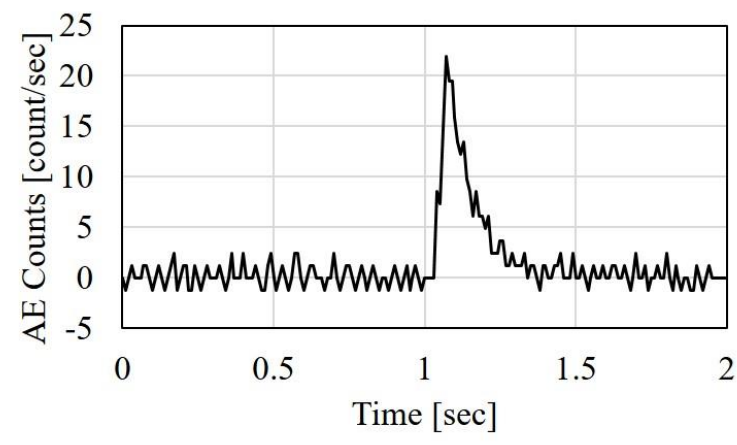

(c) point of break

Fig. 9 AE signal count. (a) overall data, (b) data at the time of plastic deformation around the peak stress, and (c) data at the point of break.
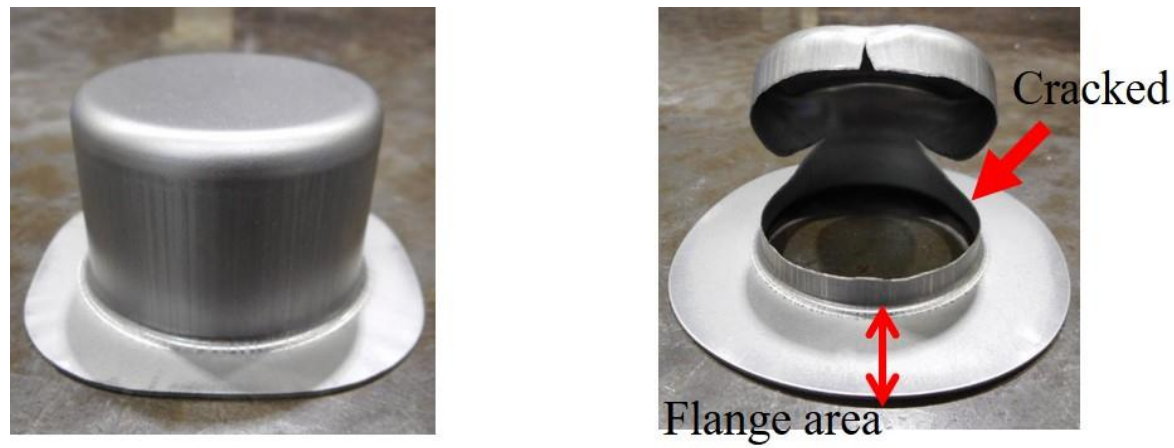

Fig. 10 The processed product obtained by the deep drawing testing. (a) normal product and (b) cracked product. 
the initial stage of the forming. Figure 11 shows the load curves during deep drawing. In Fig. 11 the vertical and horizontal axes show punch load and crank angle, respectively. In the area A, the die contacts the sheet metal. In the area $\mathrm{A}^{\prime}$, the die is coming down and forming is proceeding. In the area $\mathrm{A}^{\prime \prime}$, the die is coming up and forming finish. In the area B, the punch plate and the cushion plate contact. In Fig. 12 the vertical and horizontal axes show the count rate and axis time. In addition, Fig. 12 (a) (b) shows the normal products and Fig. 12 (c) (d) the products with cracks. Two peaks A and B exist for both the normal product and the product with cracks in Fig. 12. Each peak means that $\mathrm{A}$ is due to the contact between the die and work piece, and B due to the instantaneous deformation caused by the contact between the punch plate and the cushion plate. There are additional peaks A' and A" in Fig. 12 (c), which do not exist for the normal product

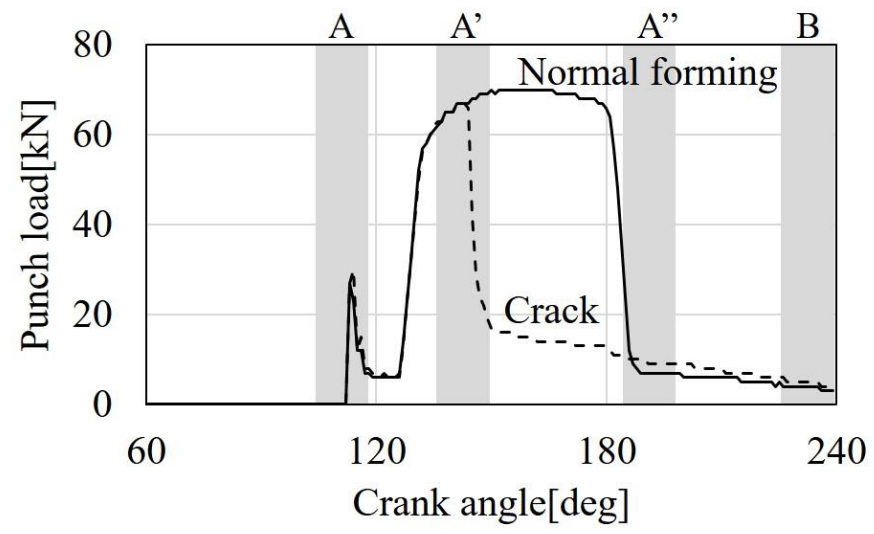

Fig. 11 Load curves during deep drawing.

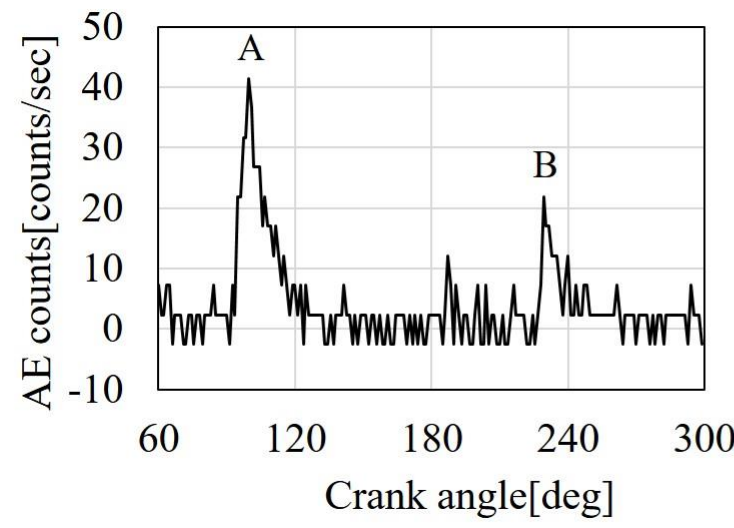

(a) normal forming

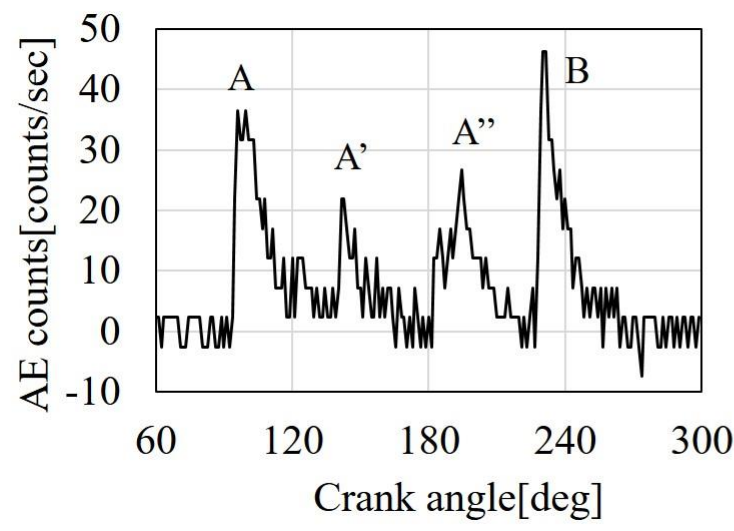

(c) crack

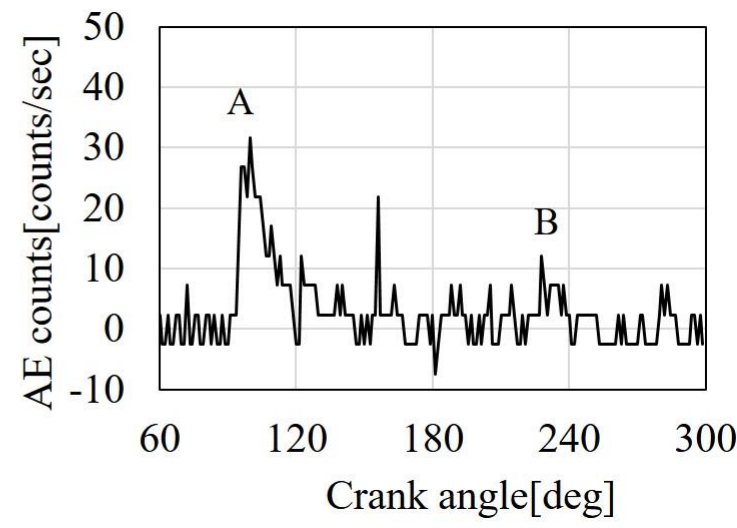

(b) normal forming

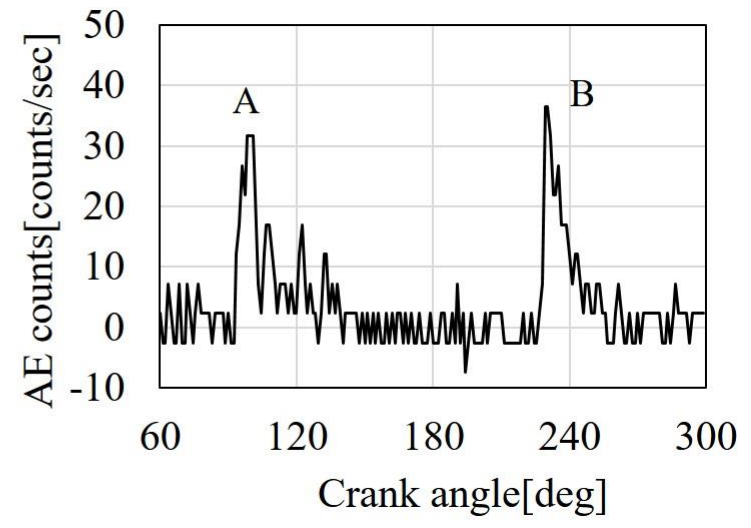

(d) crack

Fig. 12 AE signal count. (a), (b) normal forming data and (c), (d) crack. 
in Fig. 12 (a). These phenomena indicate that the additional peaks in Fig. 12 (c) are caused by the occurrence of cracks. From the change of the load in Fig. 11, the point A' is considered to be due to cracking of the material. Furthermore, it is considered that the point A" is caused by the fact that the product deformed by cracks is pushed out to the knock out (Fig. 4) while rubbing the die strongly. The peaks A' and A" are caused by the high friction due to the insufficient lubrication and by cracking of the material. Namely, these peaks give a reason of the breaks occurring during the deep drawing. Therefore, it is possible to recognize the forming state by using the present AE signal measurement system. However, the peak may appear between A and B even in normal forming as in Fig. 12 (b), or the peak may not appear between A and B even if cracking occurs as in Fig. 12 (d). Therefore, although AE count rate contains information effective for recognizing the forming state, it can be said that the conventional method is not adequate to distinguish cracks.

\subsection{Recognition of deep drawing state by machine learning}

As shown above, forming state recognition could be confirmed. Therefore, recognition through machine learning was tested. Figure 13 shows the recognition rate by combination of the activation function and the optimizer. These were the averaged values of tests performed three times. The horizontal axis is the number of nodes and the vertical axis is the recognition rate, respectively. The optimizer tends to have high recognition rate when Adam is used. The highest value is $97.3 \%$ based on the combination with the activation function tanh. SGD has the lowest recognition rate in combination with all activation functions. The recognition rate tends to be low when using sigmoid as the activation function. Figure 14 shows in detail the recognition rate by combinations with good results. The horizontal axis is the number of nodes and the vertical axis is the recognition rate, respectively. Number of hidden layer is one. These were the averaged values of tests performed three times, similarly. Error bars show the maximum and minimum values. In general, the recognition rate tends to be higher as the number of nodes increases. This is likely because features can be expressed more finely as the number of nodes becomes larger.

Figure 15 shows the recognition rate obtained by a combination of the activation functions of tanh and the

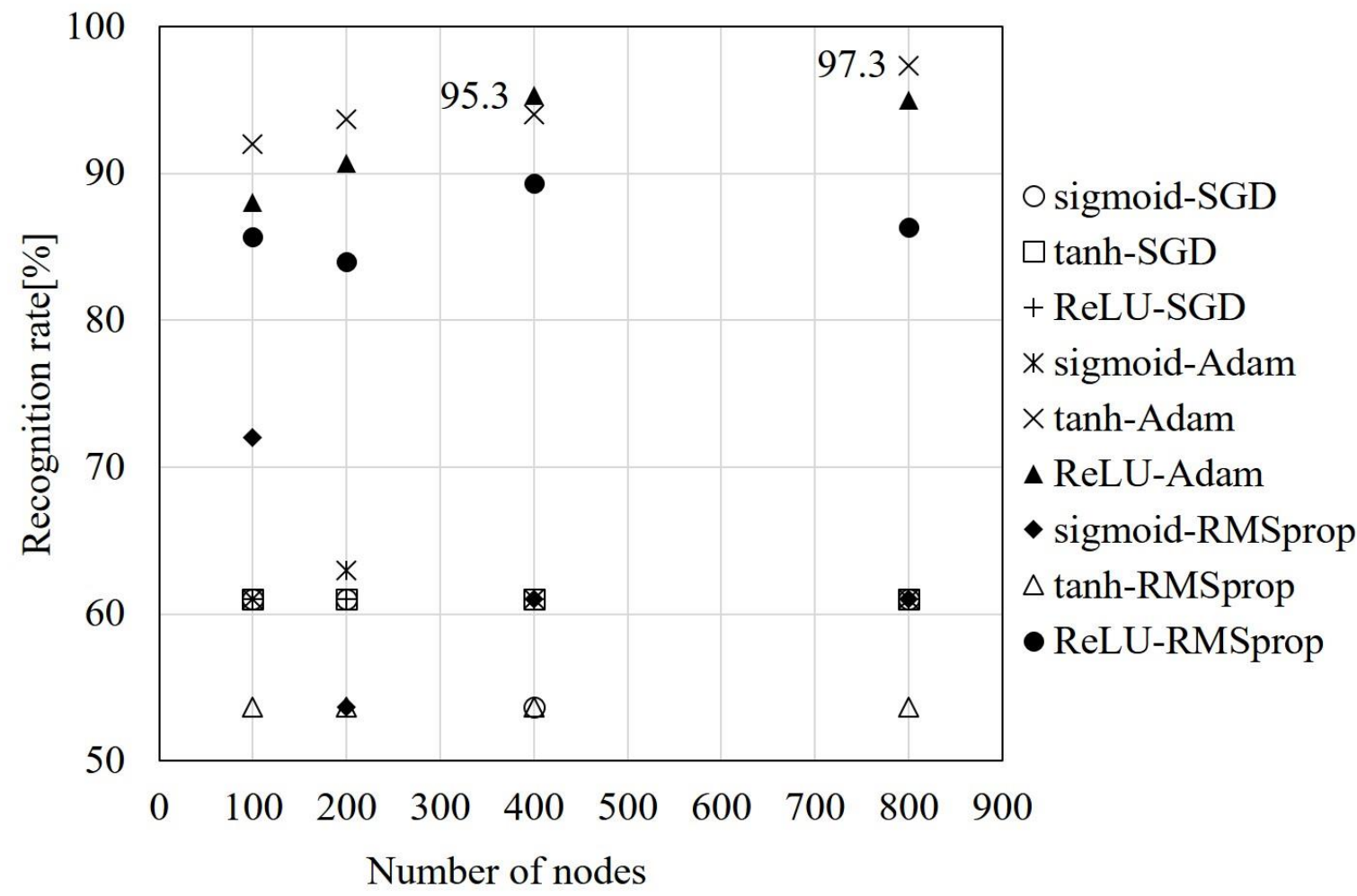

Fig. 13 Recognition rate of forming state. This is the recognition rate by combination of the optimizer and the activation function, and the recognition rate was the average value performed three times. 
optimization methods of Adam (tanh-Adam), which has the highest recognition rate as shown in Fig. 14 (tanh-Adam). Figure 15 shows the effect of the number of hidden layers on the recognition rate. Number of hidden layers of two and three are chosen for comparison. The recognition rate is the highest when the number of nodes is 200 in the three layers. The recognition rate tends to decrease with increase in the number of layers.

As shown above, the optimizer proposed by the improvement of the conventional method are effective for the collected AE data. Although the current recognition rate in this paper has not reached $100 \%$, further improvement in recognition rate is expected as learning progresses by applying the method here to the mass production site and accumulating greater number of data.

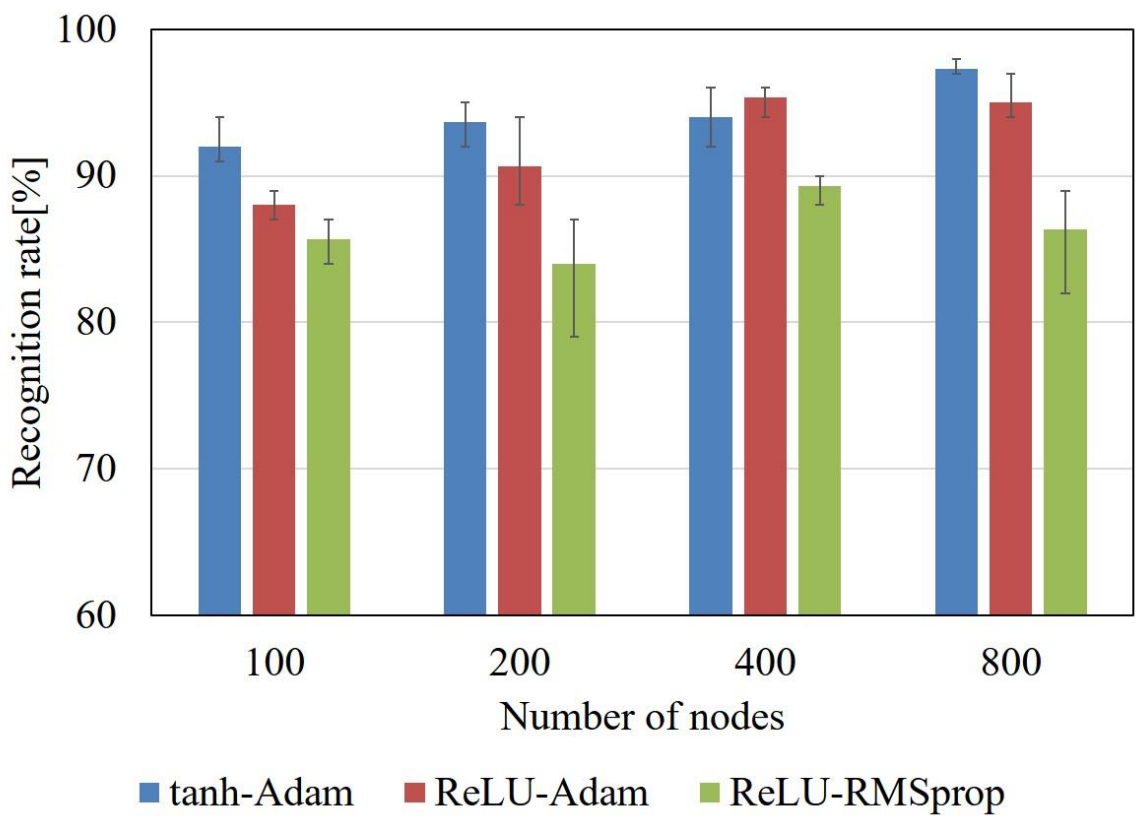

Fig. 14 Recognition rate of forming state. This is the recognition rate by combination of the optimizer and the activation function, and the recognition rate was the average value performed three times. Results of experiments with combination of the optimizer and the activation function having a recognition rate of $80 \%$ or more are shown.

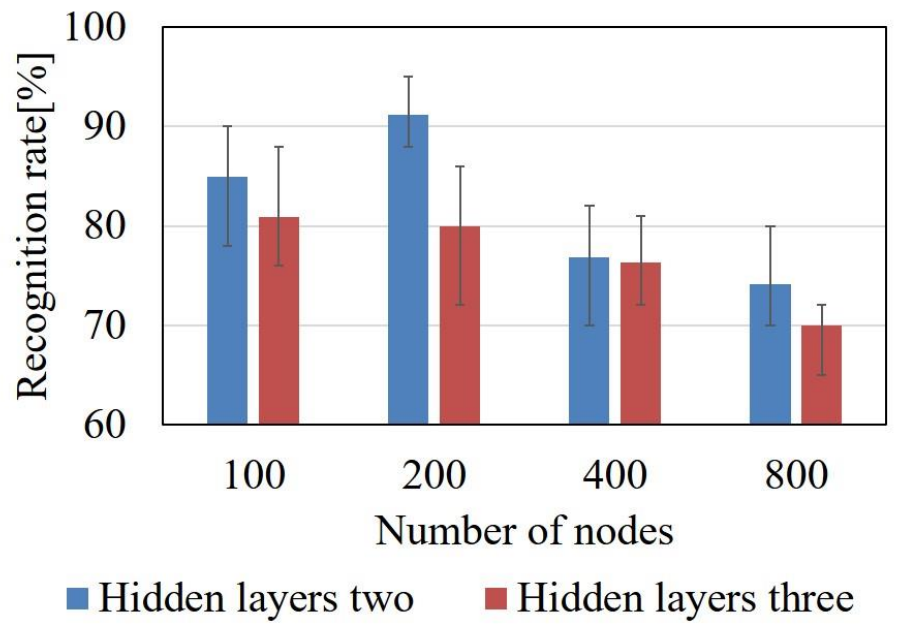

Fig. 15 Recognition rate of tanh-Adam. The recognition rates were the average values of ten times. Error bars show the maximum and minimum values. 


\section{Conclusion}

In this paper, a new processing method was proposed by using the count rate of AE signals. Machine learning, which is a multilayered neural network, is implemented to recognize defects during deep drawing processing. As a result, the following conclusions were obtained:

(1) $\mathrm{AE}$ measurements during the tensile tests showed that continuous $\mathrm{AE}$ signals are dominant during plastic deformation and material breaking leads to short bursts of AE signals.

(2) It was confirmed, especially, that there is a clear difference between the AE count generated during the plastic deformation and the point of break.

(3) The AE count during deep drawing showed that there are characteristic peaks caused by breaks occurring during the deep drawing. Therefore, it is possible to recognize the forming state during the deep drawing by measuring the AE count.

(4) Performing the forming state recognition experiment using the AE data and the forming state recognition system by multilayer neural network (deep learning) showed that the recognition rate was as high as $97.3 \%$ (tanh-Adam).

(5) Examining the deep learning parameters for AE data during deep drawing showed that the recognition rate tends to increase slightly as the number of hidden layers increases and the recognition rate decreases when the number of nodes in the hidden layer becomes too large.

(6) Recognition rate exceeding $90 \%$ was obtained for machine learning despite the small number of data used. Recognition rate improvement can be expected by accumulating more data through application to mass production sites.

\section{References}

Behrens, B., Hubner, S., and Wolki, K., Acoustic emission-A promising and challenging technique for process monitoring in sheet metal forming, Journal of Manufacturing Processes, 29 (2017), pp.281-288.

Ding, X., and He, Q., Energy-Fluctuated Multiscale Feature Learning with Deep ConvNet for Intelligent Spindle Bearing Fault Diagnosis, IEEE Transactions on Instrumentation and Measurement, Vol.66, No8(2017), pp.1926-1935.

Garcia, C., Artificial intelligence applied to automatic supervision, diagnosis and control in sheet metal stamping processes, Journal of Materials Processing Technology, 164-165 (2005), pp.1351-1357.

Hagino, N., Endou, J., Katoh, S. and Ishihama, M., Reflection and Transmission Characteristics of Ultrasonic Waves in Flat Surface Die -Visualization of Contact Conditions between Die and Workpiece During Stamping I-, Journal of the Japan Society for Technology of Plasticity, Vol.54, No.632 (2013), pp.48-52 (in Japanese).

Hagino, N., Komiya, S., Endo, J. and Ishihama, M., In-process Monitoring of Stamping Process of Thin Plate, Journal of the Japan Society for Technology of Plasticity, Vol.57, No.669 (2016), pp.983-990 (in Japanese).

Hao, S., Ramalingam, S., and Klamecki, B.E., Acoustic emission monitoring of sheet metal forming: characterization of the transducer, the work material and the process, Journal of Materials Processing Technology, 101(2000), pp.124136.

Hatano, H., Acoustic Emission: Its Fundamentals and Application, Journal of the Japan Society of Applied Physics, Vol.44, No.6 (1975), pp.564-576 (in Japanese).

Kakinoki, R., Segawa, Y., Marumo, Y., Inamura, Y., Nonaka, T. and Sakata, Y., Evaluation of Wrinkling States Using Inprocess Ultrasonic Examination during Sheet Metal Forming, Journal of the Japan Society for Technology of Plasticity, Vol.58, No.676 (2017), pp.49-53 (in Japanese).

Keitmann-Curdes, O., Hansen, C., Knoll, P., Meier, H., and Ermert, H., Ultrasonic imaging of sheet metal forming, Ultrasonics, 42 (2004), pp.989-992.

Kishi, T., Katoh, A., Kuribayashi, K., and Horiuchi, R., Amplitude Distribution of Acoustic Emission Signals in Plastic 
Deformation, Journal of the Japan Institute of Metals and Materials, Vol.41, No.9 (1977), pp.897-904 (in Japanese). Kitayama, S., Hamano, S., Yamazaki, K., Kubo, T., Nishikawa, H., and Kinoshita, H., The Square Cup Deep Drawing by Variable Blank Holder Force, Transactions of the Japan Society of Mechanical Engineers, Series C, Vol. 76, No. 766 (2010), pp. 1617-1626 (in Japanese).

Kitayama, S., Yokoyama, M., Kawamoto, K. Noda, T., Miyasaka, T., and Echigo, Y, Design optimization of variable blank holder force trajectory and slide velocity in sheet metal forming, Transactions of the JSME (in Japanese), Vol. 84, No. 863 (2018), pp.2693-2703, DOI:10.1299/transjsme.18-00162.

Koyama, J., and Yang, M., Development of Die-embedded Micro-sensing System, Journal of the Japan Society for Technology of Plasticity, Vol.51, No.596 (2010), pp.60-64 (in Japanese).

Lal, R.K., Choubey, V.K., Dwivedi, J.P., and Kumar, S., Study of factors affecting Springback in Sheet Metal Forming and Deep Drawing Process, Materials Today: Proceedings, Vol. 5 (2018), pp.4353-4358, DOI:10.1016/j.matpr.2017.12.002

Madokoro, H., Shimoi, N., Sato, K., and Xu, L., Human Sensing for Sleep Monitoring Using Film-Load Sensors, Journal of the Japan Society for Fuzzy Theory and Intelligent Informatics, Vol.28, No.6 (2016), pp.963-973 (in Japanese).

Mahayotsanun, N., Sah, S., Cao, J., Peshkin, M., Gao, R. and Wang, C., Tooling-integrated sensing systems for stamping process monitoring, International Journal of Machine Tools \& Manufacture, 49 (2009), pp.634-644.

Masuda, K., Matsui, T., Fukui, D., Fukui, K., and Machimura, T., Bat Species Classification by Echolocation Call Using a Machine Learning System, Journal of the Mammalogical Society of Japan, Vol.57, No.1 (2017), pp.19-33 (in Japanese).

Muller, T., Meyer, M., Lenske, A., Hauptmanna, M., and Majschaket, J.-P., Optical inline quality assessment of deepdrawn paperboard containers, Journal of Materials Processing Technology, Vol. 262(2018), pp.615-621, DOI:10.1016/j.jmatprotec.2018.08.003

Nishimoto, S., and Shinke, N., Evaluation of Burr During Punching Process Using AE Method, Journal of the Japan Society for Non-Destructive Inspection, Vol.54, No.10 (2005), pp.557-561 (in Japanese).

Pham, T.B., Prakash, I., and Bui, T.D., Spatial Prediction of Landslides Using a Hybrid Machine Learning Approach Based on Random Subspace and Classification and Regression Trees, Geomorphology, 303(2018), pp.256-270.

Rodrigues, D.M., Leitao, C., and Menezes, L.F., A multi-step analysis for determining admissible blank-holder forces in deep-drawing operations. Materials and Design, Vol. 31(2010), pp.1475-1481, DOI:10.1016/j.matdes.2009.08.028

Tamai, Y., Inazumi, T., and Manabe, K., Prediction of Effective Press Motion Control to Improve Deep-Drawability of Steel Sheets with FE Forming Analysis, Journal of the Japan Society for Technology of Plasticity, Vol.55, No.636 (2014), pp.34-38 (in Japanese).

Yang, M., Manabe, K., Hayashi, K., Miyazaki, M., and Aikawa, N., Data fusion of distributed AE sensors for the detection of friction sources during press forming, Journal of Materials Processing Technology, 139 (2003), pp.368-372.

Yoo, H., Liu, Z., Quang, N., Kim, S., and Bien, F., Implementation of Nondestructive Crack Detection System for Automotive Press Panel, IEEE Sensors Journal, Vol.16, No.2 (2016), pp.383-389. 